In a recent review by Meadow, ${ }^{2} 12$ of 19 cases of Munchausen syndrome by proxy involved falsified findings in the urine. Five of these 12 were cases of glycosuria. The use of ascorbic acid to identify the origin of urine specimens is simple, safe, discreet, and rapid. Ascorbic acid can be added to orange juice and given without being noticed by the patient or his caretaker. We used N-multistix-C, a reagent strip for urinalysis. One of the reagent areas tests for ascorbic acid and is based upon the chemical reduction of methylene green to its leuco form by ascorbic acid. The reagent area seems blue because of an inactive red background dye, but the colour changes from blue to purple with increasing ascorbic acid concentration.

Without supplementation, the usual amount of ascorbic acid excreted in urine is 2 to $10 \mathrm{mg} / \mathrm{dl}^{3}{ }^{3}$ Urine values of habitual users of large quantities of ascorbic acid may, however, rise to $200 \mathrm{mg} / \mathrm{dl}^{5}{ }^{5}$ The $\mathrm{N}$-multistix-C test has a sensitivity of $25 \mathrm{mg} / \mathrm{dl}$. High values of bilirubin or $\mathrm{pH}$ higher than 7.5 may cause an atypical colour. The test is insensitive to urate creatinine, gentisic acid, and salicylate.

In summary, factitious diabetes mellitus fabricated by this patient's mother was rapidly and safely confirmed by using oral ascorbic acid as a marker of the origin of the urine. The results of this objective method were presented as evidence to the judicial authorities and led to the placement of the patient in a safer environment.

\section{References}

1 Mayefsky JH, Sarnaik AP, Postellon DC. Factitious hypoglycemia. Pediatrics 1982;69:804-5.

2 Meadow R. Munchausen syndrome by proxy. Arch Dis Child 1982;57:92-8.

3 Angel J, Alfred B, Leichter J, Lee M, Marchant L. Effect of oral administration of large quantities of ascorbic acid on blood levels and urinary excretion of ascorbic acid in healthy men. Int $J$ Vitam Nutr Res 1975;45:237.

4 Atkins GL, Dean PM, Griffin WJ, Watts RWE. Quantitative aspects of ascorbic acid metabolism in man. $J$ Biol Chem 1964;239:2975.

5 Garry PJ, Owen GM, Lashley DW, Ford PC. Automated analysis of plasma and whole blood ascorbic acid. Clin Biochem 1974;7:131.

The opinions and assertions expressed herein are those of the authors and are not to be construed as official or as reflecting the views of the Navy Department or of the Department of Defense of the United States government.

Correspondence to Dr John Nading, Department of Pediatrics, Uniformed Services University of the Health Sciences, 4301 Jones Bridge Road, Bethesda, MD 20814 USA.

Received 5 November 1983

In the UK the ascorbic acid detector strip is available as C-Stix marketed by the Ames Division of Miles Laboratories-Ed.

\title{
Diabetes and developing knowledge of the body
}

\author{
C EISER, D PATTERSON, AND J H TRIPP
}

Department of Psychology, University of Exeter, and Paediatric Research Unit, Royal Devon and Exeter Hospital, Exeter

SUMMARY Fifty seven diabetic children were compared with healthy children for knowledge of their bodies and causes of diabetes. Diabetic children were more likely to include the pancreas and less likely to include the brain or stomach in drawing their bodies. The data have implications for understanding how illness affects psychological development.

Recent research suggests that lay people have poor knowledge of body organisation and function, ${ }^{1}$ and that this limits successful communication between doctor and patient. Other work indicates that illness itself may influence a patient's knowledge of the body. ${ }^{2}$ There have, however, been few empirical attempts to investigate how illness affects a child's knowledge of his body and one exception is a study of children with heart disease by Green and Levitt. ${ }^{4}$ In addition, some evidence that children with diabetes have distorted self images was reported by Kaufman and Hersher, ${ }^{5}$ who studied five teenage diabetics. It was concluded that these children believed their stomachs were larger than average (since they are required to eat more often than others they must have larger stomachs to fill), and that some thought that the pancreas was missing, either in whole or in part. Misconceptions seemed to persist despite instructions to the contrary and Kaufman and Hersher inferred that the education of diabetic children may well be hampered by their distorted knowledge of their bodies.

Our purpose was to investigate for a larger sample of diabetic children any such differences in body knowledge and associated distortions in beliefs about body functioning and the cause of diabetes. 


\section{Method}

Subjects. Fifty seven children with diabetes were contacted. These children constituted $76 \%$ of the children being cared for at the Royal Devon and Exeter Hospital, and $80 \%$ of the children being cared for in North Devon Hospital at Barnstaple. The parents of 18 other children either refused permission for their child to take part or did not reply. The chronological age of the children was mean 12 years (range 6 to 17 years). The mean age at diagnosis was $6 \cdot 7$ years (range 1 to 14 years). Each child with diabetes was matched with a healthy child for age, sex, social background, and IQ estimated by teachers.

Procedure. The children were asked to name as many parts of the insides of their bodies as they could. They were then given an outline drawing of a human body, and asked to draw what it looked like inside. On a second outline of the body, they were to draw a circle to represent the position of the following: heart, brain, stomach, lungs, liver, kidney, pancreas, and intestine. The children were also asked the function of each of these organs. Finally, they were asked if they knew what 'diabetes' was and how it was caused.

\section{Results}

Number and type of body parts. The average number of body parts mentioned by the children increased with age $(\mathrm{F}=7 \cdot 0, \mathrm{P}<0 \cdot 02)$ but did not differ between diabetics and controls (see Table 1). There were differences in terms of which body parts were drawn. Diabetics were less likely than controls to draw either a brain $\left(\chi^{2}=5.07, \mathrm{P}<\cdot 05\right)$ or a stomach $\left(\chi^{2}=8.08, P<0.01\right)$. Diabetics were more likely to draw a pancreas $\left(\chi^{2}=18 \cdot 50, \mathrm{P}<0 \cdot 001\right)$.

Function of body parts. Children's responses were coded according to Gellert. ${ }^{2}$ A series of $\chi^{2}$ tests were then conducted on the type of explanation of function offered by diabetic and healthy children. Only one of these reached significance-children with diabetes were more likely to offer an accurate explanation of the function of the pancreas $\left(\chi^{2}=17 \cdot 10, P<0 \cdot 001\right)$.

Table 1 Mean number of body parts drawn, by age

\begin{tabular}{llll}
\hline & $6-10$ yrs & $11-13$ yrs & $14-17$ yrs \\
\hline Diabetic group & 2.20 & 2.64 & 5.76 \\
Control group & 2.86 & 4.52 & 6.82 \\
\hline
\end{tabular}

Table 2 Children's explanations of the cause of diabetes

\begin{tabular}{lll}
\hline Cause & Diabetic group & Control group \\
\hline 'It just happens' & $31 \cdot 0$ & $16 \cdot 0$ \\
Shock & $4 \cdot 8$ & 0 \\
Hereditary & $21 \cdot 4$ & $56 \cdot 0$ \\
Punishment & $7 \cdot 1$ & 0 \\
Illness/infection & $21 \cdot 4$ & $4 \cdot 0$ \\
Too much sugar & $14 \cdot 3$ & $24 \cdot 0$ \\
\hline
\end{tabular}

Size of stomach in children's drawings. In $50.9 \%$ of cases, the stomach drawn by the diabetic child was clearly larger, in $31.6 \%$ of cases the stomach drawn by the healthy child was clearly larger, and for $17.5 \%$ of cases, there were no differences in size.

Definitions of diabetes and explanations of its cause. Children's explanations for the cause of diabetes were coded as follows: (1) unknown, (2) shock, (3) hereditary, (4) punishment, (5) infection, and (6) eating too much sugar. Significantly more of the children with diabetes offered some explanation (42 children with diabetes offered an explanation compared with 25 controls $\left.\chi^{2}=8 \cdot 97, \mathrm{P}<0 \cdot 01\right)$. As shown in Table 2, the type of causes given differed between the groups. Diabetic children were significantly more likely to state that the cause of the illness was unknown $\left(\chi^{2}=4.42, P<0.05\right)$. In explaining what diabetes is, diabetics were more likely than control children to give an explanation in terms of a failure of the pancreas or insulin production $\left(\chi^{2}=34.63\right.$, $P<0.001)$, while control children were more likely to offer an explanation in terms of 'eating too much sugar' $\left(\chi^{2}=12 \cdot 34, P<0 \cdot 001\right)$.

\section{Discussion}

Our purpose was to investigate how chronic illness might influence a child's knowledge of the body. We did not find evidence for distortions such as those reported by Kaufman and Hersher. ${ }^{5}$ The children with diabetes did not differ much from control children in quantitative or qualitative aspects of knowledge about their bodies. There were no differences between the groups in the number of internal body parts the children named, or in their explanations of the function of most body parts. The overall level of children's knowledge did not differ, and we found little evidence (from their drawings) that children with diabetes associated their illness with greatly enlarged stomachs.

The apparent comparability between children with diabetes and controls should not mask those aspects of the data suggesting important differences between the groups. The body parts drawn by the children in free drawings differed in type. As in 
previous studies with ill subjects, children with diabetes were more likely to include the pancreas. They were also significantly less likely to draw the brain or stomach than the healthy group. The drawings of the diabetic children who did not include the brain were not inferior in any other respect to those of their healthy controls. Their responses to questions about the function of the body organs also indicated that they knew they had a brain and why. Unfortunately, it was not possible to go back and ask children why they had originally omitted to draw the brain, but in the one case where this was possible, the girl (aged 15 years) said that what was in her head seemed of secondary importance to what was in her 'insides'. Perhaps this might lead one to speculate that diabetic and healthy children assign differences in value to various body parts. These data suggest that although there are few absolute differences between diabetic and healthy children in knowledge of their bodies, there may well be differences in attitude so that diabetics see some parts of their bodies as more or less important than healthy children.

Diabetic and control children attributed diabetes to different things. The control group favoured explanations like heredity, and the result of eating too much sugar. In contrast, paediatricians might feel heartened to learn that the types of explanations offered by the diabetic child were consistent with those generally offered by the medical profession. Only a small number apportioned any self-blame for the illness-challenging the established view that chronically sick children tend to blame themselves. ${ }^{6}$

The children included in this study all seemed to be managing their disease and its impact on their lives relatively well. These results may not, however, obtain for other groups of sick children, such as those with diseases perceived to be more threatening, or those with less supportive home backgrounds. Nevertheless, the data do suggest that it is possible to suffer a relatively threatening chronic illness during childhood, and for this to have few repercussions for the development of the child's knowledge of the body.

This work was financed by a grant from the Health Education Council, London to Professor J R Eiser. We thank Dr R Orme, Dr D Kennaird, Dr L Haas, and Mr R Towne for their help with this study.

\section{References}

1 Pendleton D, Hasler J. Doctor-patient communication. London: Academic Press, 1983.

2 Gellert E. Children's beliefs about bodily illness. Presented at the meeting of the American Psychological Association, New York: 1961 .

3 Tait CD, Jr, Ascher RC. Inside-of-the-body test. Psychosom Med 1955; 17:139-48.

${ }^{4}$ Green M, Levitt EE. Constriction of the body image in children with congenital heart disease. Pediatrics 1962;29:438-41.

5 Kaufman RV, Hersher B. Body image changes in teenage diabetes. Pediatrics 1971:48:123-8.

${ }^{6}$ Blos P. Children think about illness: their concepts and belicfs. In: Gellert E, ed. Psychological aspects of pediatric care. New York and London: Grune and Stratton, 1978.

Correspondence to $\mathrm{Dr} \mathrm{C}$ Eiser, Health Behaviour Research Group, Department of Psychology, University of Excter, Washington Singer Laboratories, Exeter EX4 4QG

Received 14 September 1983

\title{
Megacystis-microcolon-intestinal hypoperistalsis syndrome
}

\author{
I VINOGRAD, P MOGLE, O Z LERNAU, AND S NISSAN
}

The Departments of Pediatric Surgery and Radiology, Hadassah University Hospital, Mount Scopus, Jerusalem, Israel

SUMMARY Two neonates with megacystismicrocolon-intestinal hypoperistalsis syndrome are described. Both were boys. The main manifestation was functional obstruction of the urinary and gastrointestinal tracts. While there is no explanation as yet for the aetiology and pathogenesis, the diagnosis is simple, the treatment symptomatic, and the outcome fatal.
Megacystis - microcolon - intestinal hypoperistalsis syndrome was first reported by Berdon. ' ${ }^{\text {Neonates }}$ with this syndrome have a distended urinary bladder and hypoperistalsis throughout the entire gastrointestinal tract. Most of the reported cases have been girls. ${ }^{1-4}$ We report two boys with this rare syndrome and discuss its diagnostic and treatment implications. 